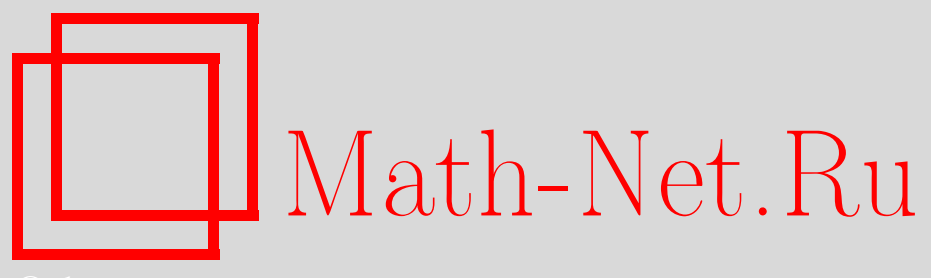

А. И. Аптекарев, В. К. Белошапка, В. И. Буслаев, В. В. Горяйнов, В. Н. Дубинин, В. А. Зорич, Н. Г. Кружилин, С. Ю. Немировский, С. Ю. Оревков, П. В. Парамонов, С. И. Пинчук, А. С. Садуллаев, А. Г. Сергеев, С. П. Суетин, А. Б. Сухов, К. Ю. Федоровский, А. К. Цих, Евгений Михайлович Чирка (к семидесятипятилетию со дня рождения), УМН, 2018, том 73, выпуск 6, 204-210

DOI: https://doi.org/10.4213/rm9864

Использование Общероссийского математического портала Math-Net.Ru подразумевает, что вы прочитали и согласны с пользовательским соглашением http://www.mathnet.ru/rus/agreement

Параметры загрузки:

IP : 3.81 .55 .215

26 апреля 2023 г., 16:27:17

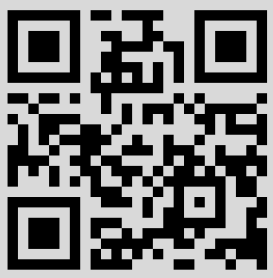




\section{Евгений Михайлович Чирка}

(к семидесятипятилетию со дня рождения)

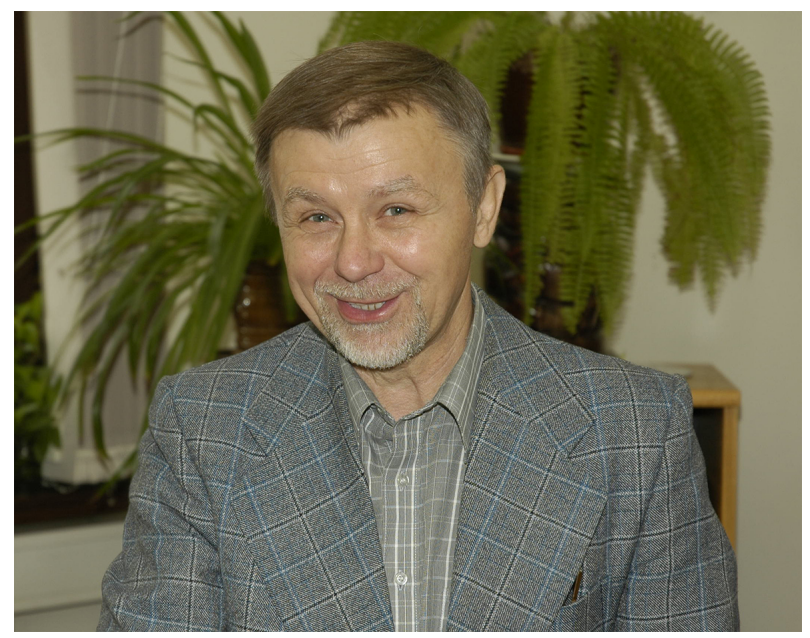

Евгений Михайлович Чирка - известный специалист в области комплексного анализа, один из основателей современной теории функций многих комплексных переменных в России.

Е. М. Чирка родился 21 октября 1942 г. в поселке Шахта 3 Снежнянского района Донецкой области в семье шахтеров.

С 1949 по 1959 г. Евгений Михайлович учился в средней школе, по окончании которой работал слесарем на строительстве ТЭЦ в Волгограде. В 1960 г. Е. М. Чирка поступил на механико-математический факультет Московского государственного университета им. М. В. Ломоносова, а после его окончания в 1965 г. был принят в аспирантуру факультета. Окончив ее в 1969 г., Евгений Михайлович был зачислен ассистентом кафедры теории функций и функционального анализа механико-математического факультета, где проработал до 1971 г. В марте 1969 г. на заседании диссертационного совета факультета он защитил кандидатскую диссертацию по теме "Приближение голоморфными функциями в $\mathbb{C}^{n}$ ".

В 1971 г. Евгений Михайлович переходит на работу в Математический институт им. В. А. Стеклова АН СССР в должности младшего научного сотрудника. В 1977 г. на заседании диссертационного совета МИАН он защищает докторскую диссертацию на тему "Аналитическое продолжение функций многих комплексных переменных и его применения", которая (как и кандидатская диссертация) была признана выдающейся.

В том же 1977 г. Е. М. Чирка был переведен в старшие научные сотрудники, а затем (в 1986 г.) - в ведущие научные сотрудники МИАН. С 2002 г. Евгений Михайлович является заведующим Отделом комплексного анализа МИАН. В 2003 г. он был избран членом-корреспондентом РАН.

DOI: https://doi.org/10.4213/rm9864 
В своих работах Евгений Михайлович неоднократно открывал новые, зачастую неожиданные закономерности, пользуясь новаторскими аналитическими и геометрическими методами, которые до него в многомерном комплексном анализе не применялись. Его исследования коснулись большинства основных направлений комплексного анализа, таких как теория приближений, граничные свойства голоморфных функций, аналитические продолжения, теория аналитических множеств, голоморфные слоения и движения, теория римановых поверхностей и пространств Тейхмюллера.

Среди наиболее известных результатов Евгения Михайловича отметим исследования, посвященные приближениям голоморфными функциями на вещественных подмногообразиях комплексных пространств.

Так, в 1969 г. он (одновременно с Р. Уэллсом и Л. Ниренбергом, но независимо от них) доказал теорему о приближении непрерывных функций голоморфными на вполне вещественных подмногообразиях в $\mathbb{C}^{n}$. В этой работе он одним из первых использовал для решения задач теории приближений $L^{2}$-методы решения $\bar{\partial}$-уравнения с оценками, незадолго до того развитые Л. Хёрмандером. Как эта техника, так и возможность аппроксимации голоморфными функциями на вполне вещественных подмногообразиях стали с тех пор общепринятыми и легли в основу многих важных результатов многомерного комплексного анализа.

В известной работе 1973 г., опубликованной в журнале "Математический сборник", Евгений Михайлович доказал практически неулучшаемые многомерные аналоги теорем Фату и Линделёфа о граничных значениях ограниченных голоморфных функций. В отличие от одномерного случая, предельные значения функций многих комплексных переменных на границе области определения существуют не только по некасательным путям, но и вдоль некоторых траекторий, касающихся границы по комплексным касательным направлениям. Этот совершенно новый феномен, исследованный Евгением Михайловичем, вошел в анналы многомерного комплексного анализа, а сформулированные им условия на допустимые касательные траектории затем многократно использовались многочисленными авторами при изучении граничных свойств различных классов голоморфных функций и отображений.

Работа 1982 г., также опубликованная в "Математическом сборнике", посвящена феномену принудительной гладкости границ одномерных аналитических множеств, лежащих в дополнении к вполне вещественным подмногообразиям. Это явление, первооткрывателем которого по праву считается Е. М. Чирка, играет важнейшую роль как в работах по классическому многомерному комплексному анализу, так и в теории псевдоголоморфных кривых Громова.

Доказанная Е. М. Чиркой в 1996 г. "обобщенная лемма Хартогса" о принудительном продолжении голоморфных функций из окрестности обобщенной фигуры Хартогса в $\mathbb{C}^{2}$ явилась одним из первых конкретных примеров применения дифференциально-топологических свойств гладких четырехмерных многообразий в комплексном анализе на пространстве $\mathbb{C}^{2}$. Подобные эффекты были в дальнейшем исследованы другими авторами с помощью теории псевдоголоморфных кривых и инвариантов Зайберга-Виттена. Использованный в его работе метод, основанный на исследовании квазилинейного $\bar{\partial}$-уравнения, был позднее применен Е. М. Чиркой для обобщения результатов Д. Салливана и З. Слодковского о продолжении голоморфных движений.

Характерной особенностью математического стиля Евгения Михайловича является использование геометрических идей для преодоления аналитических трудностей. Эта особенность ярко проявилась в его доказательстве теоремы о том, что всякий непрерывный график, разделяющий две области голоморфности в $\mathbb{C}^{n}$, расслаивается на комплексные гиперповерхности. Указанный результат, обобщающий классическую теорему Леви, был доказан Н. В. Щербиной при $n=2$, но его обобщение на многомерный случай в ситуации минимальной регулярности представлялось совершенно не очевидным. Евгению Михайловичу удалось получить такое обобщение с помощью развитой им техники голоморфных движений. 
Ставшая уже классической книга Е. М. Чирки "Комплексные аналитические множества", выпущенная издательством "Наука" в 1985 г., базируется на едином подходе к теории аналитических множеств, в основе которого лежат геометро-метрические идеи. Благодаря полноте и изяществу изложения эта книга оказала существенное влияние на всю теорию аналитических множеств. На протяжении многих лет книга оставалась практически единственным руководством по геометрической теории аналитических поверхностей для специалистов. Такой же оригинальностью изложения и элегантностью доказательств отличаются и курсы лекций "Римановы поверхности" и "Пространства Тейхмюллера", прочитанные Е. М. Чиркой в Научно-образовательном центре МИАН.

Большое влияние на развитие многомерного комплексного анализа в нашей стране оказали обзорные статьи Евгения Михайловича. Прежде всего, это обзор о граничных свойствах голоморфных функций многих переменных, написанный совместно с Г. М. Хенкиным в 1975 г. Другая обзорная статья (изданная в 1979 г. в качестве приложения к книге Р. Харви "Голоморфные цепи и их границы") посвящена применению потоков де Рама в комплексном анализе. Идеи этой статьи сыграли существенную роль в решении задачи о нормализации аналитических пространств на языке вычетных потоков и о структуре голоморфных дифференциальных форм на таких пространствах. Также следует отметить обзорные статьи Е. М. Чирки в журнале "Успехи математических наук" о геометрии CR-многообразий (1991 г.) и о голоморфных движениях и униформизации голоморфных семейств римановых поверхностей (2012 г.).

Огромную роль в развитии комплексного анализа в России сыграло активное и постоянное участие Евгения Михайловича в работе двух московских семинаров по комплексному анализу ("семинара Витушкина" в МГУ и "семинара Гончара" в МИАН), организованных в 1960-е годы А.Г. Витушкиным, А. А. Гончаром и Б. В. Шабатом. Е. М. Чирка является одним из руководителей этих семинаров уже более 20 лет. Во многом благодаря ему на семинарах царит творческая неформальная атмосфера, требующая от докладчиков и всех присутствующих максимально активного участия в обсуждениях. Евгений Михайлович щедро делится своими идеями и знаниями, оказывая тем самым большое влияние на всех участников семинаров.

Долгое время Евгений Михайлович увлекался тяжелой атлетикой (штанга) и зимним плаванием. Это увлечение спортом в прошлом помогает ему и сейчас поддерживать высокую работоспособность.

Е. М. Чирка - прекрасный человек, надежный товарищ, открытый и доступный в общении, большой оптимист по жизни.

А.И. Аптекарев, В.К. Белошапка, В.И. Буслаев, В. В. Горяйнов, В.Н. Дубинин, В.А. Зорич, Н. Г. Кружилин, С.Ю. Немировский, С.Ю. Оревков, П.В. Парамонов, С.И. Пинчук, А.С. Садуллаев, А.Г. Сергеев, С.П. Суетин, А.Б. Сухов, К.Ю. Федоровский, А.К. Цих

\section{Список научных публикаций Е. М. Чирки}

[1] "Приближение непрерывных функций голоморфными на жордановых дугах в $C^{n}$ ", Докл. АН ССCP, 167:1 (1966), 38-40; англ. пер.: "Approximation of continuous functions by functions holomorphic on Jordan $\operatorname{arcs}$ in $C^{n}$ ", Soviet Math. Dokl., 7 (1966), 336-338.

[2] "Приближения непрерывных функций голоморфными на жордановых дугах в $C^{n}$ ", Современные проблемы теории аналитических функиий, Международная конференция (Ереван, 1965), Наука, М., 1966, 324-326.

[3] Приближение голоморфными функииями в $\mathbb{C}^{n}$, Дисс. ... канд. физ.-матем. наук, Моск. гос. ун-т им. М. В. Ломоносова, М., 1968, 105 с. 
[4] "Приближение голоморфными функциями на гладких многообразиях в $\mathbf{C}^{n}$ ", $M a$ тем. сб., 78(120):1 (1969), 101-123; англ. пер.: "Approximation by holomorphic functions on smooth manifolds in $\mathbf{C}^{n}$ ", Math. USSR-Sb., 7:1 (1969), 95-114.

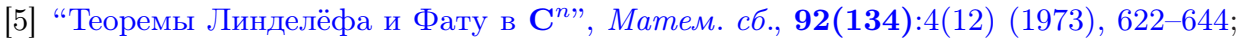

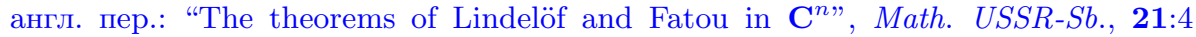
(1973), 619-639.

[6] "Приближение многочленами на звездных подмножествах $\mathbf{C}^{n}$ ", Maтем. заметки, 14:1 (1973), 55-60; англ. пер.: "Approximation by polynomials on star-shaped subsets of $\mathbf{C}^{n "}$, Math. Notes, 14:1 (1973), 586-588.

[7] "Разложения в ряды и скорость рациональных приближений для голоморфных функций с аналитическими особенностями”, Матем. сб., 93(135):2 (1974), 314-324; англ. пер.: "Expansions in series and the rate of rational approximations for holomorphic functions with analytic singularities", Math. USSR-Sb., 22:2 (1974), 323-332.

[8] "Аналитическое представление $C R$-функций", Maтем. сб., 98(140):4(12) (1975), 591-623; англ. пер.: "Analytic representation of CR-functions", Math. USSR-Sb., 27:4 (1975), 526-553.

[9] "Граничные свойства голоморфных функций нескольких комплексных переменных", Итоги науки и техн. Сер. Соврем. пробл. матем. Нов. достиж., 4, ВИНИТИ, M., 1975, 13-142 (совм. с Г. М. Хенкиным); англ. пер.: "Boundary properties of holomorphic functions of several complex variables", J. Soviet Math., 5:5 (1976), 612-687 (with G. M. Khenkin).

[10] Аналитическое продолжение функций многих комплексных переменных, Дисс. ... докт. физ.-матем. наук, Матем. ин-т им. В.А. Стеклова АН СССР, M., 1976, 246 c.

[11] "Мероморфное продолжение и скорость рациональных приближений в $\mathbf{C}^{N}$ ", $M a$ тем. сб., 99(141):4 (1976), 615-625; англ. пер.: "Meromorphic continuation and the degree of rational approximations in $\mathbf{C}^{N}$ ", Math. USSR-Sb., 28:4 (1976), 553-561.

[12] "Рациональные приближения голоморфных функций с особенностями конечного порядка", Матем. сб., 100(142):1(5) (1976), 137-155; англ. пер.: "Rational approximations of holomorphic functions with singularities of finite order", Math. USSR-Sb., 29:1 (1976), 123-138.

[13] "Аналитическое продолжение функций многих комплексных переменных и его применения", Матем. заметки, 24:3 (1978), 433-443; англ. пер.: "Analytic continuation of functions of several complex variables and its applications", Math. Notes, 24:3 (1978), 732-737.

[14] "Регуляризация и $\bar{\partial}$-гомотопия на комплексном многообразии", Докл. АН CCCP, 244:2 (1979), 300-303; англ. пер.: "Regularization and $\bar{\partial}$-homotopy on a complex manifold", Soviet Math. Dokl., 20 (1979), 73-76.

[15] "Об устранимых особенностях аналитических множеств", Докл. АН CCCP, 248:1 (1979), 47-50; англ. пер.: "On removable singularities of analytic sets", Soviet Math. Dokl., 20 (1979), 965-968.

[16] "Регулярность границ аналитических множеств", Матем. сб., 117(159):3 (1982), 291-336; англ. пер.: "Regularity of the boundaries of analytic sets", Math. USSR-Sb., 45:3 (1983), 291-335.

[17] "Комплексные аналитические множества", Комплексный анализ - многие переменные - 1, Итоги науки и техн. Сер. Соврем. пробл. матем. Фундам. направления, 7, ВИНИТИ, М., 1985, 125-166; англ. пер.: "Complex analytic sets", Several complex variables I. Introduction to complex analysis, Encyclopaedia Math. Sci., 7, Springer, Berlin, 1990, 117-158. 
[18] Комплексные аналитические множества, Наука, М., 1985, 272 с.; англ. пер.: Complex analytic sets, Math. Appl. (Soviet Ser.), 46, Kluwer Acad. Publ., Dordrecht, 1989, xx+372 pp.

[19] "О продолжении функций с полярными особенностями”, Матем. сб., 132(174):3 (1987), 383-390 (совм. с А.С. Садуллаевым); англ. пер.: "On continuation of functions with polar singularities", Math. USSR-Sb., 60:2 (1988), 377-384 (with A. Sadullaev).

[20] "О принципе симметрии для аналитических множеств", Изв. АН СССР. Сер. матем., 52:1 (1988), 200-211 (совм. с С.И. Пинчуком); англ. пер.: "On the reflection principle for analytic sets", Math. USSR-Izv., 32:1 (1989), 205-216 (with S. I. Pinchuk).

[21] "Многообразия Коши-Римана. Основные определения и некоторые результаты", Комплексный анализ и математическая физика (Дивногорск, 1987), Ин-т физики СО АН СССР, Красноярск, 1988, 125-144.

[22] "О продолжении плюриполярных особых множеств", Теория чисел, алгебра, математический анализ и их приложения, Сборник статей. Посвящается 100-летию со дня рождения Ивана Матвеевича Виноградова, Тр. МИАН, 200, Наука, M., 1991, 336-340; англ. пер.: "On the extension of pluripolar singular sets", Proc. Steklov Inst. Math., 200 (1993), 369-373.

[23] "Введение в геометрию $C R$-многообразий”, УMH, 46:1(277) (1991), 81-164; англ. пер.: "Introduction to the geometry of $C R$-manifolds", Russian Math. Surveys, 46:1 (1991), 95-197.

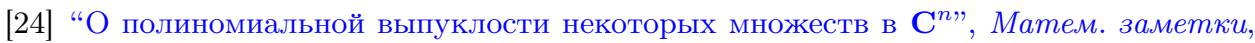
50:5 (1991), 81-89 (совм. с М. М. Смирновым); англ. пер.: "Polynomial convexity of some sets in $\mathbf{C}^{n "}$, Math. Notes, 50:5 (1991), 1151-1157 (with M. M. Smirnov).

[25] "Теорема Радо для CR-отображений гиперповерхностей”, Матем. сб., 185:6 (1994), 125-144; англ. пер.: "Radó's theorem for CR-mappings of hypersurfaces", Russian Acad. Sci. Sb. Math., 82:1 (1995), 243-259.

[26] "Normal and tangent ranks of CR mappings", Duke Math. J., 76:2 (1994), 417-431 (with C. Rea).

[27] "Removable singularities in the boundary", Contributions to complex analysis and analytic geometry, Aspects Math., E26, Friedr. Vieweg, Braunschweig, 1994, 43-104 (with E. L. Stout).

[28] "A Kontinuitätssatz", Topics in complex analysis (Warsaw, 1992), Banach Center Publ., 31, Polish Acad. Sci. Inst. Math., Warsaw, 1995, 143-150 (with E. L. Stout).

[29] "Pseudoconvexity of rigid domains and foliations of hulls of graphs", Ann. Scuola Norm. Sup. Pisa Cl. Sci. (4), 22:4 (1995), 707-735 (with N. V. Shcherbina).

[30] "On the removable singularities for meromorphic mappings", Publ. Mat., 40:1 (1996), 229-232.

[31] "On the uniform approximation on totally real sets in $\mathbf{C}^{n}$ ", Complex methods in approximation theory (Almería, 1995), Monogr. Cienc. Tecnol., 2, Univ. Almería, Almería, 1997, 23-31.

[32] "Differentiable CR mappings and CR orbits", Duke Math. J., 94:2 (1998), 325-340 (with C. Rea).

[33] "Remarks on the proof of a generalized Hartogs lemma", Complex analysis and applications (Warsaw, 1997), Ann. Polon. Math., 70 (1998), 43-47 (with J.-P. Rosay).

[34] "On boundary regularity of analytic discs", Michigan Math. J., 46:2 (1999), 271-279 (with B. Coupet, A. B. Sukhov). 
[35] “Теоремы Леви и Трепро для непрерывных графиков", Аналитические и геометрические вопросы комплексного анализа, Сборник статей. K 70-летию со дня рождения академика Анатолия Георгиевича Витушкина, Тр. МИАН, 235, Наука, МАИК "Наука/Интерпериодика", M., 2001, 272-287; англ. пер.: "Levi and Trépreau theorems for continuous graphs", Proc. Steklov Inst. Math., 235 (2001), 261-276.

[36] “Обобщенная лемма Гартогса и нелинейное $\bar{\partial}$-уравнение”, Комплексный анализ в современной математике, Фазис, М., 2001, 19-31.

[37] "Голоморфные слоения псевдовогнутых графиков", Докл. РАН, 377:4 (2001), 452-454; англ. пер.: "Holomorphic fiberings of pseudoconcave graphs", Dokl. Math., 63:2 (2001), 212-214.

[38] "A generalization of Radó's theorem", Proceedings of conference on complex analysis (Bielsko-Biała, 2001), Ann. Polon. Math., 80 (2003), 109-112.

[39] "On the removal of subharmonic singularities of plurisubharmonic functions", Proceedings of conference on complex analysis (Bielsko-Biała, 2001), Ann. Polon. Math., 80 (2003), 113-116.

[40] "О распространении голоморфных движений”, Докл. РАН, 397:1 (2004), 37-40; англ. пер.: "On the propagation of holomorphic motions", Dokl. Math., 70:1 (2004), $516-519$.

[41] "F. and M. Riesz theorem for CR functions", Math. Z., 250:1 (2005), 1-6 (with C. Rea).

[42] "Вариации теоремы Гартогса", Комплексный анализ и приложения, Сборник статей, Тр. МИАН, 253, Наука, МАИК "Наука/Интерпериодика", М., 2006, 232-240; англ. пер.: "Variations of Hartogs' theorem", Proc. Steklov Inst. Math., 253 (2006), $212-220$.

[43] "Римановы поверхности", Лекц. курсы НОЦ, 1, МИАН, М., 2006, 3-105.

[44] "On nonimbeddability of Hartogs figures into complex manifolds", Bull. Soc. Math. France, 134:2 (2006), 261-267 (with S. Ivashkovich).

[45] "Some problems in complex analysis in several variables", Узбекский матем. журн., 2009, № 1, 23-27.

[46] "Пространства Тейхмюллера", Лекц. курсы НОЦ, 15, МИАН, М., 2010, 3-150.

[47] "Неравенства Харнака, расстояния Кобаяши и голоморфные движения", Аналитические и геометрические вопросы комплексного анализа, Сборник статей, Тр. МИАН, 279, МАИК "Наука/Интерпериодика", М., 2012, 206-218; англ. пер.: "Harnack inequalities, Kobayashi distances and holomorphic motions", Proc. Steklov Inst. Math., 279 (2012), 194-206.

[48] "Голоморфные движения и униформизация голоморфных семейств римановых поверхностей", УМH, 67:6(408) (2012), 125-202; англ. пер.: "Holomorphic motions and uniformization of holomorphic families of Riemann surfaces", Russian Math. Surveys, 67:6 (2012), 1091-1165.

[49] "О $\bar{\partial}$-проблеме с $L^{2}$-оценками на римановой поверхности”, Современные проблемъ математики, механики и математической физики, Сборник статей, Тр. МИАН, 290, МАИК "Наука/Интерпериодика", М., 2015, 280-292; англ. пер.: "On the $\bar{\partial}$-problem with $L^{2}$-estimates on a Riemann surface", Proc. Steklov Inst. Math., 290:1 (2015), 264-276.

[50] "Об устранимых особенностях голоморфных функций”, Матем. сб., 207:9 (2016), 161-170; англ. пер.: "Removable singularities of holomorphic functions", Sb. Math., 207:9 (2016), 1335-1343. 
[51] "Аппроксимации Эрмита-Паде для мероморфных функций на компактной римановой поверхности”, УМН, 72:4(436) (2017), 95-130 (совм. с А. В. Комловым, P. В. Пальвелевым, С. П. Суетиным); англ. пер.: "Hermite-Padé approximants for meromorphic functions on a compact Riemann surface", Russian Math. Surveys, 72:4 (2017), 671-706 (with A. V. Komlov, R. V. Palvelev, S. P. Suetin).

[52] "Об устранимых особенностях комплексных аналитических множеств", Матем. сб., 208:7 (2017), 172-186; англ. пер.: "On removable singularities of complex analytic sets", Sb. Math., 208:7 (2017), 1073-1086.

[53] "Потенциалы на компактной римановой поверхности", Комплексный анализ, математическая физика и приложения, Сборник статей, Тр. МИАН, 301, МАИК "Наука/Интерпериодика", М., 2018, 287-319; англ. пер.: "Potentials on a compact Riemann surface", Proc. Steklov Inst. Math., 301 (2018), 272-303.

[54] “Ортогональные комплексные структуры в $\mathbb{R}^{4}$ ”, УМH, 73:1(439) (2018), 99-172; англ. пер.: "Orthogonal complex structures in $\mathbb{R}^{4}$ ", Russian Math. Surveys, 73:1 (2018), 91-159. 